\title{
$B C L 6$ gene amplification/3q27 gain is associated with unique clinicopathological characteristics among follicular lymphoma without BCL2 gene translocation
}

Kennosuke Karube ${ }^{1,6}$, Guo Ying ${ }^{2}$, Hiroyuki Tagawa ${ }^{3,4}$, Daisuke Niino ${ }^{1}$, Ryosuke Aoki ${ }^{1}$, Yoshizo Kimura ${ }^{1}$, Keiko Hashikawa ${ }^{1}$, Nobuko Suefuji ${ }^{1}$, Yasuo Sugita ${ }^{1}$, Yuko Nomura ${ }^{5}$, Kay Shimizu ${ }^{1}$, Shirou Yoshida ${ }^{1}$, Masao Seto ${ }^{3,4}$ and Koichi Ohshima ${ }^{1}$

${ }^{1}$ Department of Pathology, School of Medicine, Kurume University, Kurume, Japan; ${ }^{2}$ Department of Pathology, State Key Laboratory of Cancer Biology, Xijing Hospital, Fourth Military Medical University, Xi'an, Shannxi, People's Republic of China; ${ }^{3}$ Division of Molecular Medicine, Aichi Cancer Center Institute, Nagoya, Japan; ${ }^{4}$ Division of Hematology and Cell Therapy, Aichi Cancer Center Institute, Nagoya, Japan and ${ }^{5}$ Department of Pediatrics, School of Medicine, Fukuoka University, Fukuoka, Japan

\begin{abstract}
Although approximately 10-20\% cases of follicular lymphoma lack BCL2 gene rearrangement, there are few reports having described the alternative genetic aberrations and their association about clinicopathological features. In this study, analysis by Fluorescence in situ hybridization of BCL6 gene aberrations in 100 follicular lymphoma cases without IGH/BCL2 rearrangement resulted in the identification of four subgroups. Group I: $B C L 6$ gene rearrangement $(n=41)$; Group II: $B C L 6$ gene amplification/3q27 gain $(n=30)$; Group III: the absence of both $(n=23)$; and Group IV: the presence of both $(n=6)$. Group II showed higher grade morphology (Grade 3a/b: 93\%), higher bcl2 and MUM1 expression (73 and 57\%, respectively), and more frequent combination with BCL2 gene amplification/18q21 gain (90\%) than the other groups. BCL6 gene aberration, especially amplification/3q27 gain, indicates the presence of certain morphological and phenotypical findings in follicular lymphoma cases without IGH/BCL2 rearrangement.
\end{abstract}

Modern Pathology (2008) 21, 973-978; doi:10.1038/modpathol.2008.75; published online 23 May 2008

Keywords: follicular lymphoma; BCL2; BCL6; 3q27; MUM1

Follicular lymphoma is the most prevalent form of low-grade B-cell lymphoma in adults. ${ }^{1}$ $\mathrm{t}(14 ; 18)(\mathrm{q} 32 ; \mathrm{q} 21)$, the $B C L 2$ oncogene rearrangement accompanied by constitutive expression of the bcl-2 protein, characterizes most cases of follicular lymphoma and is a critical event in lymphomagenesis. ${ }^{2}$ However, approximately $10-20 \%$ cases of follicular lymphoma lack BCL2 rearrangement. ${ }^{1,3}$ Recent reports have described some alternative genetic aberrations for $\mathrm{t}(14 ; 18)(\mathrm{q} 32 ; \mathrm{q} 21)$-negative follicular lymphoma, for example, BCL6 gene rearrangement ${ }^{3-8}$ and trisomy $18 .{ }^{9}$ We previously performed a high-resolution bacterial artificial chromosome

Correspondence: Dr K Karube, MD, Department of Pathology, School of Medicine, Kurume University, Asahimachi 67, Kurume 830-0011, Japan.

E-mail: karube1975@yahoo.co.jp

${ }^{6}$ JSPS Research Fellow.

Received 08 January 2008; revised 21 March 2008; accepted 24 March 2008; published online 23 May 2008
(BAC) array CGH for $24 \mathrm{t}(14: 18)$-negative follicular lymphoma cases. ${ }^{10}$ We detected trisomy 3 , which is genomically characterized by gain/amplification of 18/18q, in part of t(14:18)-negative follicular lymphoma, and this aberration was mutually exclusive with BCL6 rearrangement. Furthermore, CD10(-)MUM1 $(+)$ phenotype, which suggests that this subtype may be derived from late germinal center B cells, ${ }^{10,11}$ was frequently observed in follicular lymphoma with trisomy 3 . These findings suggest that follicular lymphoma with BCL6 rearrangement and follicular lymphoma with trisomy 3 (including BCL6 gene) form a unique subgroup in follicular lymphoma without $B C L 2 / I G H$ rearrangement. But there has been no clinicopathological study to compare these subgroups of follicular lymphoma without BCL2/IGH rearrangement.

In this study, we divided follicular lymphoma without $B C L 2 / I G H$ rearrangement into four genetical subtypes based on BCL6 gene aberration status and compared their clinicopathological features. 


\section{Materials and methods}

\section{Biological Material}

Tissue specimens were obtained from human samples filed at the Department of Pathology of Kurume University between 2000 and 2007. Paraffin-embedded tissues were available in all cases. Cell suspensions were used for fluorescent in situ hybridization. These studies were agreed to by the patients or their legal guardians, whereas appropriate written consent was obtained from each participating institute or hospital.

\section{Morphological Criteria}

Follicular lymphoma was diagnosed according to new WHO classification with at least a partially follicular pattern. ${ }^{1}$ Three experienced pathologist (KK, GY, and OK) reviewed all cases. If the case had more than $50 \%$ of diffuse area, we defined these cases as follicular lymphoma with diffuse area. In some cases, (1) follicular structures are not definitive; (2) lymphoma cells showed abundant clear cytoplasm and slightly indented nuclei, namely 'marginal zone differentiation;' and (3) lymphoma cells showed predominant marginal zone invasion surrounding lymph follicles rather than follicular invasion. We deleted such cases from study because it is difficult to differentiate them from nodal marginal zone lymphoma.

\section{Immunohistochemistry}

Paraffin sections from each sample were immunostained with monoclonal antibodies against CD20 (DAKO, Glostrup, Denmark), CD3 (Novocastra, Newcastle, UK), CD10 (Novocastra), Bcl2 (DAKO), MUM1 (DAKO), and Bcl6 (Novocastra) with a previously reported method. ${ }^{12}$ Two categories were defined as negative $(<30 \%$ positively stained tumor cells) and positive $(\geqslant 30 \%$ positively stained tumor cells).

\section{Cytogenetical Analysis and Fluorescence In Situ Hybridization}

The usual G-banding method was used for cytogenetic analysis. Karyotypes were described according to the International System for Human Cytogenetics Nomenclature (1995).

Cell suspensions fixed with methanol/acetic acid (3:1) were hybridized with the LSI IgH Spectrum Green/LSI Bcl2 Spectrum Orange Dual-Fusion Translocation Probe (Vysis, Downers Grove, IL, USA) and the LSI Bcl6 Dual Color Breakpoint Probe (Vysis). Analyses were performed with previously described methods. ${ }^{13}$

Briefly, the fluorescence in situ hybridization (FISH) patterns were interpreted after counting 200 nuclei. A specimen was classified as abnormal and consistent with $I G H / B C L 2$ fusion, BCL6 break, extra copies of BCL2 gene, or extra copies of BCL6 gene, when the number of nuclei observed with these abnormal patterns was $>5 \%$.

\section{Statistical Analysis}

Student $t$-test and $\chi^{2}$ test were used to compare clinical and pathological findings among the four groups. A $P$-value of less than 0.05 represents a statistically significant difference.

\section{Results}

\section{Clinicopathological Features of Follicular Lymphoma without BCL2 Rearrangement}

Of all 609 follicular lymphoma cases enrolled, 519 were analyzed with G-banding and FISH using the $B C L 2 / I g H$ probe. Sixteen cases, already analyzed in previous study, were included ${ }^{11}$ (Table 1). The presence of $\mathrm{t}(14 ; 18)(\mathrm{q} 32 ; \mathrm{q} 21)$ detected by G-banding or IGH/BCL2 with FISH rearrangement resulted in the exclusion of 387 cases as follicular lymphoma with BCL2 gene rearrangement. Of the 132 follicular lymphoma cases without BCL2 rearrangement as evidenced by not only G-banding but also FISH, 100 cases with sufficient materials were suitable for further analysis. All 100 cases were confirmed as the primary diagnostic biopsies. No secondary biopsies or recurrences after therapy were included. Fiftynine cases were successfully analyzed by G-banding method (Supplementary File 1). Additional analysis of all cases of follicular lymphoma without BCL2 rearrangement for $B C L 6$ gene aberrations by FISH showed that 41 cases featured only $B C L 6$ rearrangement (Figure 2a) and 30 cases only BCL6 amplification/3q27 gain (Figure 2b). Six cases possessed both BCL6 abnormalities and 23 did not possess either aberration. This indicates that the simultaneous occurrence of BCL6 rearrangement and BCL6 amplification/3q27 gain is relatively rare. Of the 36 cases with BCL6 amplification/3q27 gain, 33 possessed three or four signals in one lymphoma cell. Although three cases had more than four signals, two of them, which were analyzed by G-banding also, showed obvious amplification of chromosomes, including chromosome 3 (case nos. 27 and 29, Supplementary File 1). Although we could not confirm the chromosomal duplication by using centrometric probes, these findings indicate that BCL6 amplification/3q27 gain reflects chromosome 3 duplication, including chromosome 3 trisomy, rather than tandem $B C L 6$ gene amplification. As for BCL6, 32 of the 36 cases with BCL2 amplification/ 18q21 gain were found to possess chromosome 18 duplication, and not tandem BCL2 gene amplification (data not shown). 
Table 1 shows the clinicopathological features of follicular lymphoma without BCL2/IgH rearrangement. Morphologically, 60 cases (60\%) showed Grade 3a/b (3a: 33\% (33 cases), 3b: 27\% (27 cases)), whereas immunohistochemically, $56 \%$ were found to be positive for CD10 and $42 \%$ for bcl2.

\section{Genetic Subgroup of Follicular Lymphoma without BCL2 Rearrangement}

We divided follicular lymphoma without BCL2 rearrangement into four subgroups: Group I: $B C L 6$ gene rearrangement/3q27 translocation $(n=41)$; Group II: BCL6 gene amplification/3q27 gain $(n=30)$; Group III: the absence of both $(n=23)$; and Group IV: the presence of both $(n=6)$. Most of the cases were assigned to Groups I, II, and III, and relatively few to Group IV. BCL2 amplification/ 18 q21 gain was detected in no less than $90 \%$ of Group II, but only 7.3 and $8.6 \%$, respectively, each of Groups I and III possessed this aberration $(P<0.001)$. This finding proves the strong relationship between BCL6 amplification/3q27 gain and $B C L 2$ amplification/18q21 gain. We summarized the cytogenetic abnormalities focusing on chromosomal abnormalities associated with gain in 59 cases with G-band data to rule out the possibility that this relationship was the result from the widespread tendency for chromosomal gain as an underlying mechanism (Supplementary File 1). To identify the specific abnormalities and avoid the secondary changes, the stemline karyotype (the simplest abnormal clone) was used as the representative karyotype for all analyses. In patients who had composite karyotypes or when stemline karyotypes could not be specified, we used the karyotype that were most encountered. As shown in Table 2, Group II had more abnormalities associated with gain compared with Groups I and III (average: Group II: 4, Group I: 1.7, and Group III: 2.1). Furthermore, the amplification associated with 3q27 and 18q32 were preferential $(21 / 52 ; 40 \%)$ rather than only a part of widespread tendency of chromosomal gain in Group II.

Morphologically, 59\% of Group I and 61\% of Group III were low grade (Grade 1 or 2), but most of Group II (93\%) was high grade (Grade 3a/b) $(P<0.001)$ (Figures 1 and 2$)$. Fourteen cases $(47 \%)$ of Group II were Grade 3b and this proportion was significantly higher than Group I (10 cases, 25\%) and Group III (0 cases) $(P<0.05)$. Immunohistochemistry showed higher bcl2 and MUM1 expression in Group II (73 and 57\%) than in Group I (29 and $25 \%)$ or Group III $(26 \%$ and $0 \%)(P<0.001)$. Follicular lymphoma with CD10-MUM1 + phenotype is included in follicular lymphoma without $B C L 2$ rearrangement and accounted for 15 cases in Group II (50\%), which is significantly higher than in Groups I and III (15 and 0\%, respectively; $P<0.005$ ). Group III showed similar pathological characteris- 
Table 2 Chromosomal changes in each group

\begin{tabular}{lrrr}
\hline $\begin{array}{l}\text { Group and number } \\
\text { of cases analyzed } \\
\text { by G-band }\end{array}$ & $\begin{array}{c}\text { Cases with any } \\
\text { chromosomal gain }\end{array}$ & $\begin{array}{c}\text { Total chromosomal gain } \\
\text { events among positive } \\
\text { cases (average per case) }\end{array}$ & $\begin{array}{c}\text { Gain including 3q27 and 18q32 } \\
\text { abnormalities among total } \\
\text { chromosomal gain events }\end{array}$ \\
\hline Group I $(n=26)$ & $9(35 \%)] 1$ & $15(1.7)] 3$ & $0(0 \%)] 5$ \\
Group II $(n=15)$ & $13(87 \%)] 21(40 \%)]$ & $3(16 \%)$ \\
Group III $(n=14)$ & $9(64 \%)$ & $52(4)$ & $4(36 \%)$ \\
Group IV $(n=4)$ & $2(50 \%)$ & $19(2.1)] 4$ & $5: P=0.003,6:$ \\
& $1: P=0.0012,2:$ & $3: P=0.003,4: P=0.02$, & $P=0.052, \chi^{2}$ test
\end{tabular}


Figure 1 Morphological features of follicular lymphomas without BCL2 gene translocation. (a, b) From a representative case of Group I and (c-e) from a case categorized into Group III. (a, c) In H\&E sections, the lymphoma cells form definite follicular structures. As shown in (b), more than half of Group I cases were diagnosed as low grade (Grade 1 or 2), whereas almost all of the Group III cases were diagnosed as high grade (Grade 3a or 3b) (c). (e) More than half of Group III cases were positive for MUM1. Original magnification: (a, c): $\times 25,(\mathbf{b}, \mathbf{d}, \mathbf{e}): \times 200$.

tics to those of Group I. The ratios of high-grade morphology (39\%), BCL2 amplification/18q21 gain $(8.6 \%)$, CD10 positivity $(70 \%)$, bcl2 positivity $(26 \%)$, and bcl6 positivity (87\%) were not significantly different from those in Group I, but MUM1 positivity was different (MUM1: 0 vs $25 \%, P<0.01$ ). Group IV had only a small number of cases $(n=6)$, indicating BCL6 gene rearrangement and amplification/3q27 gain was uncommon. Therefore, definite comparisons with other groups were difficult.
Clinical information about male/female ratio and age was collected for all cases, and other clinical findings (B symptoms (89 cases), LDH level (79 cases), performance status (46 cases), and clinical stage (57 cases)) were partly obtained.

There were generally no significant differences between clinical features among groups. However, Group III showed relatively favorable clinical features as well as infrequent occurrence of $\mathrm{LDH}$ elevation (5\%) and B symptom (5\%) compared with 


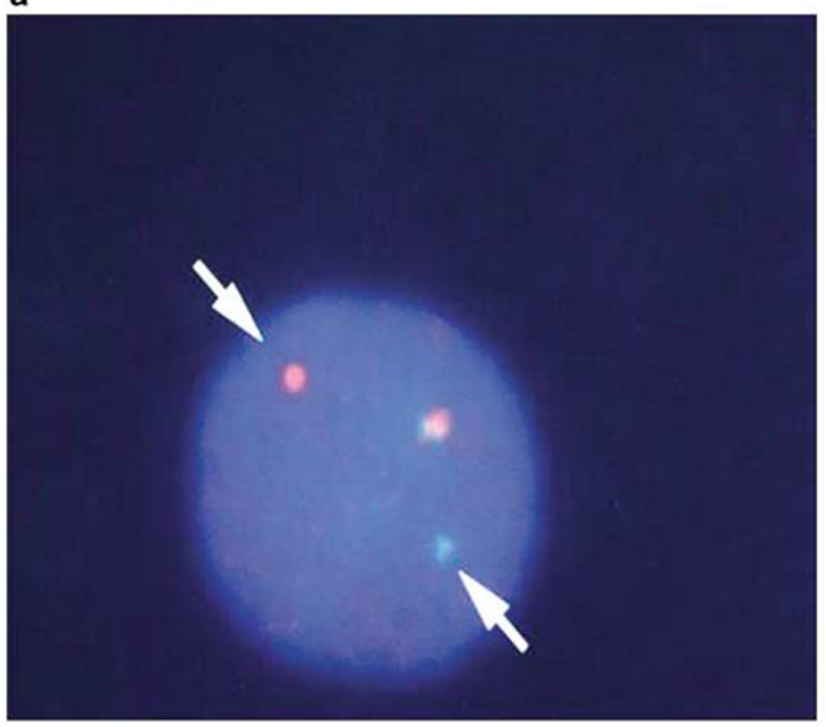

b

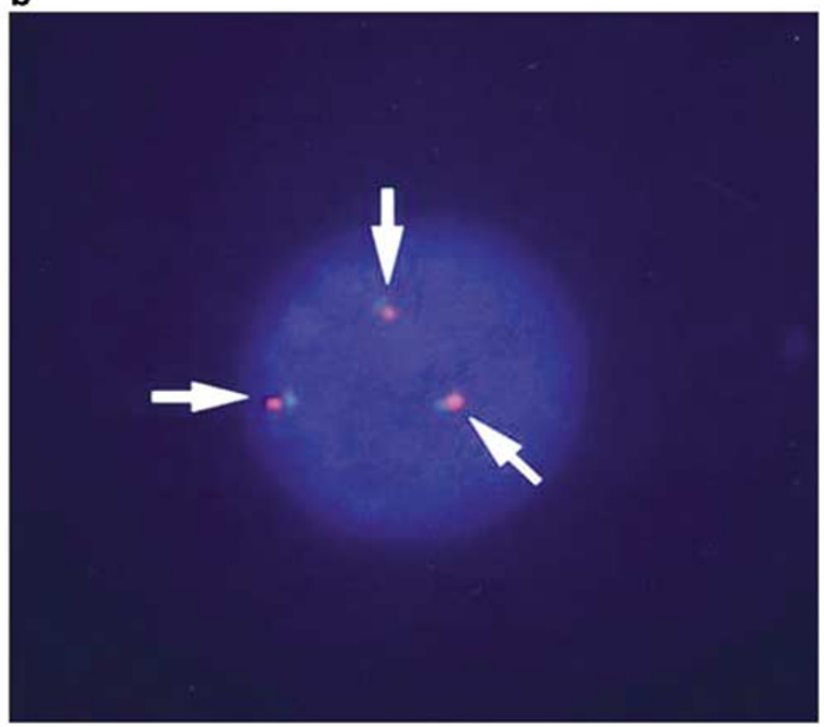

Figure 2 Representative result of dual-color FISH analysis for BCL6 (3q27). Rearrangement (a) and extra copies (b) of BCL6 (3q27) are detected.

other major groups (LDH, Groups I and II: 20 and $17 \%$; B symptom, Groups I and II: $22 \%(P=0.08)$ and $33 \%(P=0.02))$. Group III contained relatively few advanced stage cases (Stage III/IV: 29\%) compared with other groups (Groups I and II: 58 and $71 \%)$. Only Groups II and III showed a significant difference $(P=0.02)$.

\section{Discussion}

In this study, we performed morphological, immunohistochemical, and genetical review of follicular lymphoma without BCL2 rearrangement. Follicular lymphoma without $B C L 2$ rearrangement showed high-grade morphology and relatively low CD10 and bcl2 expression compared with follicular lymphoma with BCL2 rearrangement. These findings are compatible with those of previous reports including our study. ${ }^{3,4,9-11,14}$ In such cases, marginal zone lymphoma with follicular colonization and marginal zone lymphoma with vague nodular pattern were considered as differential diagnosis. But we confirmed they are follicular lymphoma because (1) all cases were lymph node lesion, not extranodal sites, (2) all of them showed distinct follicular growth pattern, (3) and lymphoma cells are composed of medium-sized cells with angulated, elongated, twisted, or cleaved nuclei and scant pale cytoplasm known as 'centrocytes,' and large transformed cells with round or oval nuclei, vesicular chromatin, and a narrow rim of cytoplasm known as 'centroblasts,' which are morphological hallmark of follicular lymphoma.

In follicular lymphoma with BCL2 rearrangement, the $B C L 2$ oncogene is relocated into an immunoglobulin gene locus, leading to deregulated expression of the bcl2 protein. This rearrangement causes abrogation of apoptosis, and it is considered to be the initial etiological mutation. ${ }^{2,15,16}$ In contrast, it has not been determined which genetic aberration is the etiological trigger of lymphomagenesis in follicular lymphoma without $B C L 2$ rearrangement. $B C L 6$ gene translocation is a representative gene aberration in follicular lymphoma without BCL2 rearrangement. $^{3,4,17}$ Previous studies have reported that follicular lymphoma with BCL6 rearrangement is frequently observed in high-grade (Grade 3 ) follicular lymphoma cases. ${ }^{3,7}$ In our study, however, more than half of the follicular lymphoma cases with BCL6 rearrangement (59\%) showed low-grade morphology, whereas its morphological and phenotypical features, especially in the bcl6 protein, were not significantly different from those of follicular lymphoma cases without any BCL6 gene aberration (Group III). These findings indicate that the rearrangement of the BCL6 gene in follicular lymphoma may not perform as important a role, especially in transformation, as previously reported. Interestingly, a recent study on IgH $S$ region break points in $\mathrm{t}(3 ; 14)(\mathrm{q} 27 ; \mathrm{q} 32)(B C L 6 / \operatorname{IgH})$ demonstrated that in follicular lymphoma most IgH break points involved $\mathrm{S} \gamma$, whereas in diffuse large B-cell lymphoma mostly $\mathrm{S} \mu$-regions are involved. The $\mathrm{S} \gamma$-break point in follicular lymphoma implies that bcl6 expression is driven by an $\mathrm{I} \gamma$-promoter whose activation requires CD40 and cytokine signaling, whereas the $\mathrm{S} \mu$-break point leads to $B C L 6$ expression driven by the constitutively active intronic $\mathrm{IgH}$ enhancer. ${ }^{18}$ Another study found that the BCL6 gene rearrangement did not affect bcl6 mRNA expression. ${ }^{19}$ The significance of BCL6 gene rearrangement in follicular lymphoma, therefore, deserves to be discussed again on the basis of further evidence. 
In contrast, follicular lymphoma with BCL6 amplification/3q27 gain (Group II) showed significant pathological characteristics (high-grade morphology, BCL2 gene amplification/18q21 gain and bcl2 expression and CD10-MUM1 + phenotype ${ }^{11}$ ). In this group, BCL2 gene amplification/18q21 gain was thought to be due to trisomy 18 , which possibly implicate the effect of an increased dosage, and related to lymphomagenesis similar to that seen in follicular lymphoma with BCL2 rearrangement as previously reported..$^{9}$ Almost all cases in which Gbanding analysis was successfully performed (15 cases) showed complex chromosomal aberrations, not only trisomy 3 and 18 (Supplementary File 1), and these numerous aberrations may be involved in the transformation/high-grade morphology.

In conclusion, follicular lymphoma without BCL2 gene translocation possesses heterogenetic biological characteristics, two of which are BCL6 gene amplification/3q27 gain and BCL6 gene translocation. The former is associated with definitive clinicopathological features, whereas the latter has relatively less effect on pathological characteristics. In this study, we could not follow up the clinical prognosis of these cases, but it can be expected that analysis of prognosis will disclose more significant clinical differences among these groups.

\section{Acknowledgement}

This study was supported in part by Grants-in-Aid from the Ministry of Education, Science and Culture, Japan. KK and OK designed the study; KK executed the study and analyzed data; KK wrote the paper; and TH, SM, AR, KY, HK, SN, SY, NY, SK, YS, ND, and GY each contributed to the analysis of data.

\section{References}

1 Nathwani BN, Harris NL, Berger F, et al. Follicular lymphoma. In: Jaffe ES, Stein H, Vardiman JW (eds). Pathology and Genetics of Tumours Haematopoietic and Lymphoid Tissues. World Health Organization Classification of Tumours. IARC Press: Lyon, France, 2001, pp 162-167.

2 Hockenbery D, Nunez G, Milliman C, et al. Bcl-2 is an inner mitochondrial membrane protein that blocks programmed cell death. Nature 1990;348:334-336.

3 Guo Y, Karube K, Kawano R, et al. Low-grade follicular lymphoma with $\mathrm{t}(14 ; 18)$ presents a homogeneous disease entity otherwise the rest comprises minor groups of heterogeneous disease entities with Bcl2 amplification, Bcl6 translocation or other gene aberrances. Leukemia 2005;19:1058-1063.

4 Jardin F, Gaulard P, Buchonnet G, et al. Follicular lymphoma without $\mathrm{t}(14 ; 18)$ and with
BCL-6 rearrangement: a lymphoma subtype with distinct pathological, molecular and clinical characteristics. Leukemia 2002;16:2309-2317.

5 Bosga-Bouwer AG, van den Berg A, Haralambieva E, et al. Molecular, cytogenetic, and immunophenotypic characterization of follicular lymphoma grade 3B; a separate entity or part of the spectrum of diffuse large B-cell lymphoma or follicular lymphoma? Hum Pathol 2006;37:528-533.

6 Bosga-Bouwer AG, van Imhoff GW, Boonstra R, et al. Follicular lymphoma grade $3 \mathrm{~B}$ includes 3 cytogenetically defined subgroups with primary $\mathrm{t}(14 ; 18), 3 \mathrm{q} 27$, or other translocations: $\mathrm{t}(14 ; 18)$ and $3 \mathrm{q} 27$ are mutually exclusive. Blood 2003;101:1149-1154.

7 Katzenberger T, Ott G, Klein T, et al. Cytogenetic alterations affecting BCL6 are predominantly found in follicular lymphomas grade $3 \mathrm{~B}$ with a diffuse large Bcell component. Am J Pathol 2004;165:481-490.

8 Ott G, Katzenberger T, Lohr A, et al. Cytomorphologic, immunohistochemical, and cytogenetic profiles of follicular lymphoma: 2 types of follicular lymphoma grade 3. Blood 2002;99:3806-3812.

9 Horsman DE, Okamoto I, Ludkovski O, et al. Follicular lymphoma lacking the $\mathrm{t}(14 ; 18)(\mathrm{q} 32 ; \mathrm{q} 21)$ : identification of two disease subtypes. Br J Haematol 2003;120: 424-433.

10 Tagawa H, Karube K, Guo Y, et al. Trisomy 3 is a specific genomic aberration of $t(14 ; 18)$ negative follicular lymphoma. Leukemia 2007 21:2549-2551.

11 Karube K, Guo Y, Suzumiya J, et al. CD10-MUM1+ follicular lymphoma lacks BCL2 gene translocation and shows characteristic biologic and clinical features. Blood 2007;109:3076-3079.

12 Ohshima K, Kawasaki C, Muta H, et al. CD10 and Bcl10 expression in diffuse large B-cell lymphoma: CD10 is a marker of improved prognosis. Histopathology 2001;39:156-162.

13 Kodama T, Ohshima K, Nomura K, et al. Lymphomatous polyposis of the gastrointestinal tract, including mantle cell lymphoma, follicular lymphoma and mucosa-associated lymphoid tissue lymphoma. Histopathology 2005;47:467-478.

14 Eshoa C, Perkins S, Kampalath B, et al. Decreased CD10 expression in grade III and in interfollicular infiltrates of follicular lymphomas. Am J Clin Pathol 2001;115:862-867.

15 Tsujimoto Y, Ikegaki N, Croce CM. Characterization of the protein product of bcl-2, the gene involved in human follicular lymphoma. Oncogene 1987;2:3-7.

16 Chao DT, Korsmeyer SJ. BCL-2 family: regulators of cell death. Annu Rev Immunol 1998;16:395-419.

17 Sekiguchi N, Kobayashi Y, Yokota Y, et al. Follicular lymphoma subgrouping by fluorescence in situ hybridization analysis. Cancer Sci 2005;96:77-82.

18 Ruminy P, Jardin F, Picquenot JM, et al. Two patterns of chromosomal breakpoint locations on the immunoglobulin heavy-chain locus in B-cell lymphomas with $\mathrm{t}(3 ; 14)(\mathrm{q} 27 ; \mathrm{q} 32)$ : relevance to histology. Oncogene 2006;25:4947-4954.

19 Jardin F, Ruminy P, Bastard C, et al. The BCL6 protooncogene: a leading role during germinal center development and lymphomagenesis. Pathol Biol (Paris) 2007;55:73-83.

Supplementary Information accompanies the paper on Modern Pathology website (http://www.nature.com/ modpathol) 